\title{
Enxágue bucal com carboidrato: recurso ergogênico capaz de otimizar o desempenho físico
}

\author{
Carbohydrate mouth rinse: an ergogenic aid able of optimizing \\ the performance
}

1 Universidade Estadual de Londrina. Programa de Pós-Graduação em Educação Física. Londrina, PR. Brasil.

2 Universidade Estadual de Londrina. Grupo de Estudo e Pesquisa em Sistema Neuromuscular e Exercício. Londrina, PR. Brasil.

3 Universidade Estadual de Londrina. Grupo de Estudo e Pesquisa em Metabolismo, Nutrição e Exercício, Departamento de Educação Física. Londrina, PR. Brasil.

Recebido em 15/07/10 Revisado em 20/10/10 Aprovado em 03/11/10
Resumo - Alguns estudos têm demonstrado que a simples presença de carboidrato na boca humana pode favorecer a ativação de algumas regiões cerebrais específicas com impacto positivo para a melhoria do desempenho físico. Assim, recentemente, a estratégia de enxágue bucal com carboidrato tem sido adotada com sucesso em exercícios físicos intensos $\left(>70 \% \mathrm{VO}_{2 \max }\right)$ com duração de, aproximadamente, 60 min. Nesse sentido, o propósito deste estudo foi discutir, com base nas informações disponíveis na literatura, a eficácia ou não da estratégia de enxágue bucal com carboidrato para a melhoria do desempenho físico, bem como os possíveis mecanismos envolvidos. Os resultados produzidos, embora ainda preliminares, são bastante promissores, o que nos leva a acreditar que o enxágue bucal com carboidrato possa ser uma estratégia bastante interessante, sobretudo, pela fácil aplicabilidade, baixo custo e boa eficácia para a melhoria do desempenho físico em esforços físicos intensos e prolongados.

Palavras-chave: Enxágue bucal; Nutrição; Carboidrato; Ergogênico nutricional; Desempenho esportivo.

Abstract - Studies have shown that the mere presence of carbohydrate in the human mouth promotes the activation of some specific brain regions, with a positive impact on physical performance. Thus, the strategy of carbohydrate mouth rinse has been recently adopted with success in strenuous exercise (>70\% $\mathrm{VO}_{2 \max }$ ) lasting approximately $60 \mathrm{~min}$. In this respect, the objective of this study was to discuss, based on literature data, the efficacy of this strategy to improve physical performance, as well as the possible mechanisms involved. The results produced, although still preliminary, are promising and led us to believe that carbohydrate mouth rinse is an interesting strategy, especially because of its easy application, low cost and good efficacy in improving physical performance during intense and prolonged physical exercise.

Key words: Mouth rinse; Nutrition; Carbohydrate; Ergogenic aid; Athletic performance. 


\section{INTRODUÇÃO}

A constante busca pela melhoria do desempenho atlético tem sido um grande desafio para profissionais e pesquisadores da área do esporte, ao longo das últimas décadas. Assim, o uso de diferentes substâncias alimentares ou a adoção de estratégias nutricionais que possam postergar a fadiga e/ ou maximizar o desempenho físico tem atraído o interesse, em particular, da comunidade científica. Entre os ergogênicos nutricionais mais investigados historicamente, a partir de diferentes estratégias de consumo e de diferentes dosagens, destacam-se os carboidratos. Nesse sentido, existem evidências de que a ingestão de carboidratos $(\mathrm{CHO})$ durante esforços físicos prolongados, com duração de pelo menos duas horas, pode favorecer a melhoria do desempenho físico. Os principais mecanismos que têm sido advogados para este fenômeno são a manutenção da homeostase glicêmica e a redução da depleção das reservas de glicogênio muscular, principalmente, nas fibras do tipo I.

Por outro lado, não existe consenso de que a ingestão de $\mathrm{CHO}$ durante a prática de exercícios físicos possa melhorar o desempenho físico em atividades com, aproximadamente, uma hora de duração, embora alguns pesquisadores defendam a utilização dessa estratégia em exercícios, sobretudo, de intensidade mais alta $\left(>75 \% \mathrm{VO}_{2 \max }\right)$, como observado em ciclistas treinados para este tipo de esforço1. A falta de consenso tem sido motivada pela ausência de informações sobre mecanismos de ação robustos que pudessem justificar a adoção de tal estratégia. Alguns pesquisadores afirmam que a contribuição dessa estratégia para a taxa total de oxidação de $\mathrm{CHO}$ é muito pequena para explicar qualquer efeito sobre o desempenho físico ${ }^{2}$. Vale destacar que a ingestão de $\mathrm{CHO}$ antes do exercício pode resultar em uma hiperglicemia e hiperinsulinemia, seguida por um rápido declínio da glicemia nos primeiros minutos do exercício, um fenômeno denominado de hipoglicemia reativa. Acredita-se que este efeito hipoglicemiante possa prejudicar acentuadamente o desempenho físico, principalmente, em esforços prolongados, embora alguns estudos não tenham confirmado tal hipótese ${ }^{3}$.

Considerando que a infusão intravenosa de glicose não parece afetar o desempenho físico, a despeito de uma maior disponibilidade de glicose no plasma, como observado em atividade contra-relógio (60 min de ciclismo $)^{2}$, parece que o mecanismo pelo qual o desempenho possa ser melhorado estaria atrelado ao trato alimentar ${ }^{2}$. Na tentativa de testar essa hipótese, ciclistas treinados, após serem submetidos a apenas a um enxágue bucal com $\mathrm{CHO}$ ao invés da ingestão tradicional, apresentaram melhora significante no desempenho físico em atividade contra-relógio $(60 \mathrm{~min} \text { de ciclismo })^{4}$. Assim, parece que os sinais aferentes oriundos de receptores de $\mathrm{CHO}$, presentes na boca, poderiam desempenhar uma função importante para a melhora no desempenho físico ${ }^{4}$.

A partir desses achados tem crescido o interesse da comunidade científica em investigar os possíveis efeitos ergogênicos do enxágue bucal com $\mathrm{CHO}$ para a melhoria do desempenho físico, em diferentes exercícios físicos. Com base nas informações produzidas até o presente momento, tentaremos discutir a eficácia ou não da estratégia de enxágue bucal com carboidrato para a melhoria do desempenho físico, bem como os possíveis mecanismos que poderiam explicar um suposto efeito ergogênico.

\section{ENXÁGUE BUCAL COM CHO E MECANISMOS DE AÇÃO}

Apesar de ainda existirem poucas informações sobre os reais mecanismos pelos quais a ingestão de $\mathrm{CHO}$ poderia auxiliar a melhoria do desempenho físico, algumas hipóteses merecem ser discutidas de forma mais consistente. Inicialmente, hipotetizou-se que uma maior oferta de $\mathrm{CHO}$ no plasma induzida pela ingestão de $\mathrm{CHO}$, antes e durante o exercício, poderia auxiliar na manutenção da glicemia durante a realização de exercícios físicos, em particular, quando os estoques de glicogênio muscular e hepático fossem depletados ${ }^{5}$. Entretanto, durante exercícios físicos relativamente curtos $(\sim 1 \mathrm{~h})$ e intensos $\left(>75 \% \mathrm{VO}_{2 \max }\right)$, esse mecanismo per se não justificaria o possível efeito ergogênico da ingestão de $\mathrm{CHO}$, visto que o organismo contém glicogênio suficiente para sustentar esse nível de esforço, sem que haja hipoglicemia ${ }^{5}$. Embora um aumento na taxa de oxidação de glicose promovido pela ingestão de $\mathrm{CHO}$ pudesse ser o mecanismo básico para justificar uma possível melhoria no desempenho físico, tal hipótese não tem sido confirmada em exercícios físicos com duração de 45-50 min realizados em estado estável ${ }^{6}$.

Mais recentemente, a partir de análises por imagens de ressonância magnética funcional, foi observado que a simples presença de líquidos carboidratados na boca humana, ativa importantes regiões cerebrais relacionadas a sensações de motivação, incluindo córtex cingulado anterior, ínsula, opérculo frontal, córtex orbitofrontal e 
estriado?. Assim, um possível efeito ergogênico induzido pelo enxágue bucal com $\mathrm{CHO}$ passou a ser discutido por alguns pesquisadores, com base na ativação de regiões cerebrais que parecem estar envolvidas com a motivação e o controle motor? Adicionalmente, tais respostas parecem ocorrer, independente do sabor do líquido oferecido (nível de doçura do $\mathrm{CHO}$ ), mediante participação de receptores orais, até o momento não identificados?

O possível mecanismo de ação poderia ser descrito da seguinte forma: (1) A simples presença de um alimento na boca gera informação sensorial gustatória para o sistema nervoso central ${ }^{8}$; (2) A informação sensorial gerada nos quimiorreceptores da cavidade oral, por sua vez, estimula neurônios gustatórios de primeira ordem que se projetam para a região rostral do núcleo do trato solitário (rNTS); (3) Neurônios de segunda ordem no rNTS projetam-se para o núcleo ventral posterior medial do tálamo; (4) A partir daí, a informação é transmitida para o córtex insular.

Considerando que o córtex insular projeta neurônios para diversas regiões corticais, dentre as quais o córtex motor, acredita-se que o córtex insular pode ser uma possível rota de ativação para o córtex motor ipsilateral. Esta hipótese se baseia em achados de estudos com pacientes em recuperação de acidente vascular encefálico, nos quais o córtex insular está mais ativado do que em situações normais.

Assim, provavelmente, o enxágue bucal com $\mathrm{CHO}$ promova uma informação gustatória que percorre a via descrita, uma vez que a atividade do córtex insular parece aumentada nessas condições, bem como a atividade no opérculo frontal, córtex órbito frontal e estriado? . Vale destacar que já há algum tempo a atividade do córtex insular, do córtex orbito frontal, do córtex motor e do córtex cingulado vêm sendo relacionadas à percepção de fadiga ${ }^{9}$. Nesse sentido, Sidhu et al. ${ }^{10}$ demonstraram que o exercício de ciclismo pode acarretar reduções na capacidade do córtex motor em dirigir os músculos extensores de joelho, sugerindo que diminuição da excitabilidade do córtex motor pode ser um dos mecanismos responsáveis pela queda da produção de potência ao longo do exercício físico ${ }^{10}$. Por outro lado, ao se enxaguar a boca com uma bebida carboidratada, a excitabilidade do córtex motor foi aumentada em 30\% após exercício físico extenuante $^{11}$. Esses dados sugerem que a melhoria do desempenho físico, observada após enxágue bucal com $\mathrm{CHO}$, está associada a mecanismos centrais, provavelmente, pelo aumento da capacidade do córtex motor (facilitada pelo córtex insular) em recrutar os músculos esqueléticos para produção de potência durante esforços físicos de longa duração.

Com base nessas informações, acreditamos que um dos mecanismos pelo qual o enxágue bucal com $\mathrm{CHO}$ possa melhorar o desempenho físico seja o seguinte: ao enxaguar a boca com uma solução carboidratada, os quimiorreceptores presentes na língua e cavidade oral excitam neurônios de primeira ordem que levam a informação ao rNTS. Este, por sua vez, irá atuar sobre o núcleo ventral posterior medial do tálamo que projeta neurônios para o córtex insular aumentando a sua atividade. Uma vez ativado, o córtex insular pode estimular a excitabilidade do córtex motor, tornando-o mais eficiente para gerar estímulos motores o que faz com que a produção de potência seja aumentada durante o exercício físico, para uma mesma percepção subjetiva de esforço (Figura 1). No nosso ponto de vista, essa hipótese merece ser testada em futuros estudos.

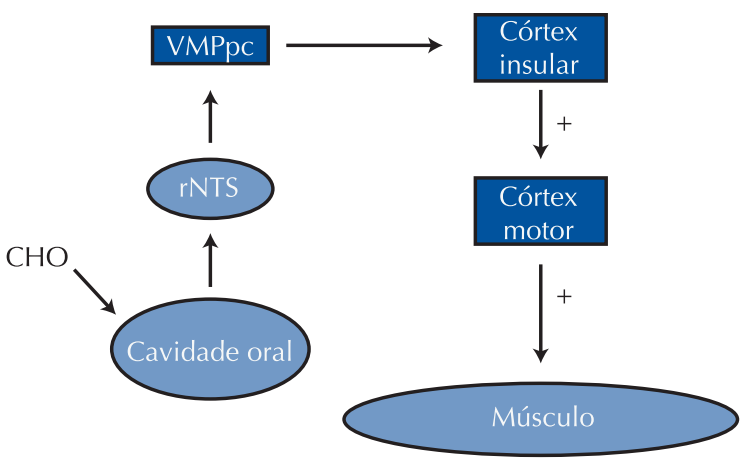

Figura 1. Mecanismo de ação do enxágue bucal com carboidrato sobre o desempenho. $\mathrm{CHO}=$ Carboidrato, $\mathrm{rNTS}=$ parte rostral do Núcleo do trato solitário, $\mathrm{VPMpc}=$ núcleo ventroposterior medial do tálamo.

\section{ENXÁGUE BUCAL COM CHO E DESEMPENHO FÍSICO}

Dentre os estudos que procuraram investigar o possível efeito ergogênico do enxágue bucal com $\mathrm{CHO}$ sobre o desempenho esportivo, a grande maioria tem utilizado como modelo de estudo o ciclismo ${ }^{4,5,7,12}$ e a corrida ${ }^{13-15}$. Vale ressaltar que dos sete artigos analisados, cinco deles $(71,4 \%)$ verificaram melhoria do desempenho em exercícios físicos de duração de aproximadamente $60 \mathrm{~min}$. De acordo com os resultados encontrados, o enxágue bucal deve ser realizado com líquidos contendo baixas concentrações de carboidrato (6-6,4\% de glicose ou maltodextrina). Os indivíduos devem ser orientados para realizarem movimentos com a língua, mantendo a bebida na boca (aproximadamente $100 \mathrm{ml}$ ) por cerca de 5-10 s. Após esse período a solução deve ser descartada. Esse procedimento deve ser realizado antes e durante 
a realização do exercício físico (aproximadamente a cada $12 \%$ de duração do esforço), de acordo com o tipo de exercício de interesse. Outra aplicação prática do enxágue bucal com $\mathrm{CHO}$ é a adoção dessa estratégia em estudos que visem desidratar os participantes durante sessões prolongadas de exercício físico no calor. Acredita-se que o enxágue bucal com $\mathrm{CHO}$ possa melhorar o desempenho físico independente das perdas hídricas, proporcionando um aumento da magnitude da desidratação.

O enxágue bucal com $\mathrm{CHO}$ pode melhorar o desempenho físico, contribuindo para que os sujeitos se tornem mais rápidos e eficientes durante as atividades, sem alterações na percepção subjetiva de esforço ${ }^{4,5,7,15}$.

\section{CONSIDERAÇÕES FINAIS}

A utilização do enxágue bucal com $\mathrm{CHO}$ em tarefas fechadas tem demonstrado melhorar o desempenho em exercício com duração de aproximadamente uma hora. Desse modo, o efeito dessa estratégia deve ser disseminado entre os técnicos e assistentes técnicos ou, ainda, entre pessoas envolvidas na preparação de atletas, para que os achados possam ser aplicados em situações práticas, envolvendo o treinamento de atletas e os resultados encontrados subsidiem a tomada de decisão mais segura sobre a adoção ou não dessa estratégia para fins competitivos.

Por fim, acreditamos que o enxágue bucal com $\mathrm{CHO}$ possa ser uma estratégia bastante interessante para a melhoria do desempenho físico de atletas de diferentes modalidades e para estudos que envolvam esforços exaustivos e mecanismos de fadiga. Os resultados produzidos, embora ainda preliminares, são bastante promissores, o que nos leva a acreditar que essa possa ser uma estratégia bastante interessante, sobretudo, pela fácil aplicabilidade, baixo custo e boa eficácia para a melhoria do desempenho físico em esforços físicos intensos e prolongados. A aplicação dessa estratégia merece ser testada mais amplamente, de modo que os mecanismos envolvidos possam ser gradativamente desvendados.

\section{Agradecimentos}

Os autores agradecem a CAPES (H.B. e M.V.C) pelas bolsas de estudo e ao CNPq (E.S.C.) pela bolsa de produtividade concedida.

\section{REFERÊNCIAS BIBLIOGRÁFICAS}

1. Jeukendrup A, Brouns F, Wagenmakers AJ, Saris, WH. Carbohydrate-electrolyte feedings improve 1 h time trial cycling performance. Int J Sports Med 1997;18(2):125-9.
2. Carter JM, Jeukendrup AE, Mann CH, Jones DA. The effect of glucose infusion on glucose kinetics during a 1-h time trial. Med Sci Sports Exerc 2004;36(9):1543-50.

3. Febbraio MA, Chiu A, Angus DJ, Arkinstall MJ, Hawley JA. Effects of carbohydrate ingestion before and during exercise on glucose kinetics and performance. J Appl Physiol 2000;89(6):2220-6.

4. Carter JM, Jeukendrup AE, Jones DA. The effect of carbohydrate mouth rinse on 1-h cycle time trial performance. Med Sci Sports Exerc 2004;36(12):2107-11.

5. Pottier A, Bouckaert J, Gilis W, Roels T, Derave W. Mouth rinse but not ingestion of a carbohydrate solution improves 1-h cycle time trial performance. Scand J Med Sci Sports 2010;20(1):105-11.

6. Below PR, Mora-Rodriguez R, Gonzalez-Alonso J, Coyle EF. Fluid and carbohydrate ingestion independently improve performance during $1 \mathrm{~h}$ of intense exercise. Med Sci Sports Exerc 1995;27(2):200-10.

7. Chambers ES, Bridge MW, Jones DA. Carbohydrate sensing in the human mouth: effects on exercise performance and brain activity. J Physiol 2009;587(Pt 8):1779-94.

8. de Araujo IE, Simon SA. The gustatory cortex and multisensory integration. Int J Obes (Lond) 2009;33 Suppl 2:S34-43.

9. St Clair Gibson A, Baden DA, Lambert MI, Lambert EV, Harley YX, Hampson D, Russell VA, Noakes TD. The conscious perception of the sensation of fatigue. Sports Med 2003;33(3):167-76.

10. Sidhu SK, Bentley DJ, Carroll TJ. Locomotor exercise induces long-lasting impairments in the capacity of the human motor cortex to voluntarily activate knee extensor muscles. J Appl Physiol 2009;106(2):556-65.

11. Gant N, Stinear CM, Byblow WD. Carbohydrate in the mouth immediately facilitates motor output. Brain Res 2010;1350:151-8.

12. Beelen M, Berghuis J, Bonaparte B, Ballak SB, Jeukendrup AE, van Loon LJ. Carbohydrate mouth rinsing in the fed state: lack of enhancement of time-trial performance. Int J Sport Nutr Exerc Metab 2009;19(4):400-9.

13. Rollo I, Cole M, Miller R, Williams C. The Influence of Mouth-Rinsing A Carbohydrate Solution on 1 Hour Running Performance. Med Sci Sports Exerc 2009.

14. Rollo I, Williams C, Gant N, Nute M. The influence of carbohydrate mouth rinse on self-selected speeds during a 30-min treadmill run. Int J Sport Nutr Exerc Metab 2008;18(6):585-600.

15. Whitham M, McKinney J. Effect of a carbohydrate mouthwash on running time-trial performance. J Sports Sci 2007;25(12):1385-92.

\section{Endereço para correspondência}

Henrique Bortolotti, Av. Garibaldi Deliberador, número 325, apto 24, bloco 4 , CEP 86050-280, Londrina, PR, Brasil.

E-mail: hbortolotti@hotmail.com 